\title{
La maladie de Hurler (mucopolysaccharidose type I, MPSI). Mutations et polymorphismes
}

L'étude de la MPSI apporte un modèle privilégié de l'évolution des idées dans la compréhension des maladies génétiques. Les MPS ont d'abord eu une classification clinique. Au cours des années 70 l'analyse biochimique permit d'identifier les déficits enzymatiques responsables; on eut alors une double surprise: d'un côté un même tableau clinique, par exemple dans la MPSIII ou maladie de Sanfilippo, pouvait relever de plusieurs déficits enzymatiques différents; à l'opposé, la plus grave de toutes, la MPSI ou maladie de Hurler, avec dysostose, dysmorphies faciale et corporelle, anomalies cardio-vasculaires, retard intellectuel, et la moins grave, la maladie de Scheie ou MPSIS, étaient toutes deux dues au déficit en la même enzyme, $\alpha$-L-iduronidase (IDUA); les dosages d'activité ne pouvaient les différencier. A la recherche d'interprétations simples, on supposa qu'il y avait deux lésions moléculaires du même gène, l'une causant la forme grave, l'autre la forme bénigne ; et les cas de gravité intermédiaires devaient être des hétérozygotes composites, avec un allèle portant chacune des mutations. On attendait avec confiance l'heure des explications apportée par l'avènement de la biologie moléculaire. L'heure venue, les résultats sont beaucoup plus complexes que prévu.

$\mathrm{m} / \mathrm{s} n^{\circ} 11$ vol. 9, novembre 93
L'ADNc puis le gène de l'IDUA ont été clonés en 1991 par Scott et al. (Adélaïde, Australie) [1]. Le gène est situé sur le bras court du chromosome 4 en 4 pl6.3 non loin du télomère. Il couvre environ $19 \mathrm{~kb}$ et se divise en 14 exons [2]. La protéine précurseur compte 653 acides aminés, et détache un peptide signal de 26 acides aminés qui libère le polypeptide de $74 \mathrm{kDa}$. Le Northern blot détecte un messager unique de 2,3 kb.

Les travaux de cette même équipe [3], et ceux d'un groupe israélien travaillant avec E. Neufeld (Los Angeles, CA, USA) [4, 5], ont défini une quinzaine de mutations, et ce nombre augmente rapidement. Certaines sont relativement fréquentes mais aucune ne prédomine à elle seule. Deux mutations rendent compte de la moitié des cas sévères de MPSIH (Hurler); ce sont des mutations non-sens, l'une dans la région N-terminale, Gln $70 \rightarrow$ Ter, l'autre plus distale, Trp $402 \rightarrow$ Ter. Dans la forme bénigne MPSIS prédominent également 2 mutations, un faux-sens Arg $89 \rightarrow$ Gln, et un changement $\mathrm{G} \rightarrow \mathrm{A}$ dans un intron entre les exons 5 et 6 . Dans l'ensemble, sans donner le détail de toutes les observations, on peut faire les remarques suivantes:

- les mutations non-sens fréquentes, avec codon de terminaison, sont souvent trouvées sous forme d'hété- rozygotes composites avec une autre, rendant compte comme prévu de certaines formes de sévérité intermédiaire ;

- quelques types de mutation mettent en jeu des mécanismes particuliers: l'une d'elles provoque une élongation de la molécule par changement d'un codon de terminaison (38 acides aminés supplémentaires, Ter $\rightarrow$ Cys). Enfin, une mutation non-sens crée, du fait du codon TAG formé (Tyr $343 \rightarrow$ Ter), un site accepteur d'épissage, 57 nucléotides en aval du site normal.

Le groupe israélien a, par ailleurs, fait une constatation importante pour la génétique des populations [4] : dans un travail portant sur des familles arabes et druzes vivant en Israël, les auteurs ont eu la surprise de constater que, dans un périmètre très restreint et une ethnie druze apparemment homogène, on comptait quatre mutants différents. C'est un exemple de plus démontrant que l'hypothèse d'un effet fondateur unique peut ne pas se vérifier à l'expérience.

Mais la nature des mutations n'est pas seule à influer sur la sévérité des symptômes. Surimposés aux mutations, de nombreux polymorphismes ont été découverts dans le gène, et un total de 17 changements de base a été détecté jusqu'à présent [3]. Huit de ces changements sont dans 
des exons, 2 entraînent un changement d'acide aminé, Gln $33 \rightarrow$ His et Ala $361 \rightarrow$ Thr. Ces polymorphismes pourraient avoir deux types de conséquences : ils rendent probablement compte de la variabilité de l'activité normale de l'IDUA, et des difficultés éprouvées lorsqu'on voulait dépister les hétérozygotes dans les familles des malades par dosage enzymatique. Plusieurs de ces polymorphismes peuvent modifier la stabilité ou l'épissage du messager. La seconde conséquence est donc d'exercer une influence sur la gravité des mutations pathogènes, qui peut être modulée par un polymorphisme déterminé. Il est admis aujourd'hui que des différences génétiques et de milieu (naguère baptisées "terrain") peuvent aider à interpréter des différences cliniques chez des malades porteurs du même génotype pathologique. L'examen des polymorphismes de l'IDUA pourrait contribuer à l'éclaircissement de tels mécanismes.

J.C.D.

1. Scott HSD, Anson DS, Orsborn AM, Nelson PV, Clements PR, Morris CP, Hopwood IJ. Human $\alpha-\mathrm{L}$-iduronidase : cDNA isolation and expression. Proc Nall Acad Sci USA 1991; 88: $965-9$.

2. Scott HS, Guo XH, Hopwood IJ, Morris CP. Structure and sequence of the human $\alpha$ L-iduronidase gene. (ienomics 1992; 13: 1.311-3.

3. Scott HS, Nelson PV, Litjens T, Hopwood IJ, Morris CP. Multiple polymorphisms within the $\alpha$-L-iduronidase gene (IDUA) : implications for a role in modification of MPSdisease phenotype. Hum Mol (ienet 1993; 2 : 1471-3.

4. Bach G, Moskowitz SM, Tieu PT, Matynia T, Neufeld EF. Molecular analysis of Hurler syndrome in Druze and Muslim Arab patients in Israel: multiple allelic mutations of the IDUA gene in a small geographic area. $\mathrm{Am}$ J Hum Genet 1993 ; 53: 330-8.

5. Tieu PT, Matynia A, Bach G, Hwang M Dion B, Neufeld EF. Novel mutations in the gene encoding alpha-L-iduronidase in mucopolysaccharidosis l (MPS I). Am / Hum (ienet 1993: 53 (suppl) : 955 (abstr).

\section{COURRIER}

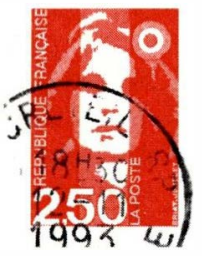

\section{L'embryologie comparée et expérimentale contre le Darwinisme}

Le «Débat" de M. Delsol et al. sur la théorie synthétique de l'évolution $\left(\mathrm{m} / \mathrm{s} n^{\circ} 10\right.$, vol. 8, p. 1079-87), bien qu'esquivant le débat (les détracteurs sont minoritaires, peu informés, et leurs reproches d'ordre philosophique) a le mérite de le relancer. Le problème est abordé par la diversité des formes dans le règne animal, mais son unité et les analogies dans les modalités évolutives des grands embranchements sont passées sous silence. Or elles supposent des complications analogues dans les modalités du développement, respectant le plan fondamental de l'organisation, ce que les mutations au hasard d'un programme génétique n'auraient pu faire, même triées par une sélection sévère. La notion de programme est elle-même douteuse (Lewin, Genes, Flammarion, p. 720), contredite par la teneur des noyaux en ADN chez les vertébrés supérieurs, égale, sinon inférieure à celle des Protistes (Grassé, L'évolution du vivant, $p$. 311-9) et par bien d'autres faits $\left(\mathrm{m} / \mathrm{s} n^{\circ} 9\right.$, vol. $2, \quad p .514$ et $\mathrm{m} / \mathrm{s}$ $n^{\circ} 3$, vol. 4, p. 168). Cette notion, par ailleurs, autorise un silence quasitotal sur l'embryologie comparée et expérimentale et sur les objections qu'elle soulève à l'encontre des principes darwiniens. En outre, bien que ses concepts classiques ne soient plus valables, cette science nous livre une somme considérable de données qui permettent de reconstituer les mécanismes évolutifs et d'en prédire les modalités, telles que les a décrites Grassé (R. Chandebois: Pour en finir avec le darwinisme, Espaces 34, Montpellier, 1993). Parce que la programmation du développement requiert une instruction particulière, par le soma, des cellules germinales femelles, la lignée évolutive apparaît comme un système cybernétique téléonomique auquel est intégré le système téléonomique "individu", qui s'organise par le jeu constructif des interactions cellulaires. Le travail du système «lignée " est de donner aux œufs des moyens accrus pour prolonger toujours davantage l'ontogenèse et donc engendrer des individus toujours plus grands et plus complexes avec la même quantité d'information génétique. La variation génotypique a créé le buissonnement des lignées en révélant les potentialités morphogénétiques multiples des systèmes «individu »

\section{Rosine Chandebois}

Université de Provence, Case 62, place Victor-Hugo, 13331 Marseille Cedex 3, France 